grad von Demokratien (ein faktischer Wiederabdruck aus PVS 3/2007) präsentiert die MOE-Erfolgsfälle als theoriewidrige Überraschung. Meines Erachtens steht seine These, das „puristisch“ handlungstheoretische „Dilemma der Gleichzeitigkeit“ sei damit widerlegt, aber auf wackligen Füßen. Laut Merkel habe dieses Theorem „deutlich weniger als 50 Prozent der Ergebnisse“ (S. 39) vorausgesagt. Dies trifft aber rechnerisch nur zu, weil lediglich 18 der 29 Länder ausgewählt werden, in denen der Staatssozialismus zusammengebrochen ist - die elf übrigen postsozialistischen Staaten werden nicht einbezogen; sie sind heute sämtlich nicht-demokratisch verfasst.

Wichtiger noch: Nimmt man die explizite Aufmerksamkeit für Kontingenz ernst, die den handlungstheoretischen Erklärungsansatz auszeichnet, kann das Dilemma-Theorem nicht mit dem ihm hier unterstellten starken Prognoseanspruch dahergekommen sein. Es postulierte die logische Unmöglichkeit, bestimmte Systemreformen gleichzeitig und endogen zum Erfolg zu führen, wenn Mechanismen der Konfliktbearbeitung, die Hinnahmebereitschaft der Transformationsverlierer und andere günstige Bedingungen nicht verfügbar wären. Wie gerade die zehn MOE-Länder zeigen, in denen das Dilemma zugunsten von Demokratie und Marktwirtschaft aufgelöst wurde, gab es in der Realität aber kaum logische Gleichzeitigkeit, hingegen viel politische Geduld, und der Demokratisierungserfolg war in einigen, für die Theorie relevanten Fällen entscheidend an starke externe Unterstützung gebunden. Dieses Argument durchzieht einige Beiträge des Sammelbands, für Frank Schimmelfennig und Guido Schwellnus ist es sogar zentral (explizit S. 288 f., S. 296). In diesem Sinne scheint Merkels These auch durch die vorliegenden empirischen Untersuchungen zumindest nicht gestärkt.

Als ganz besonderer Service für die Leser ist zu würdigen, dass der Band einen ausführlichen Materialanhang enthält, der die wichtigsten Daten für alle untersuchten Strukturdimensionen in allen zehn Ländern im Zeitraum von 1989/90 bis 2008 zur Verfügung stellt.

Petra Stykow

\title{
Schriften von Hugo Preuß als entschiedene Verstärkung liberaler Gegenperspektiven
}

Preuß, Hugo: Gesammelte Schriften. Zweiter Band: Öffentliches Recht und Rechtsphilosophie im Kaiserreich, herausgegeben und eingeleitet von Dian Schefold in Zusammenarbeit mit Christoph Müller, Tübingen 2009, 891 Seiten, € 99,-.

Das vorliegende Buch bietet ein Teilstück einer gar nicht hoch genug einzuschätzenden Gesamtedition, denn sie zeigt Hugo Preuß auf einer ersten Ebene als politischen Professor der Kaiserzeit par excellence und ist weiter geeignet, ihn als Urschöpfer ${ }^{1}$ der Weimarer Verfassung sowie seinen weniger bekannten Kampf um ihre Einhaltung verständlicher zu machen. Dabei handelt es sich, um den zuletzt veröffentlichten der fünfbändig angelegten Gesamtedition. Die beiden ersten nunmehr geschlossen vorliegenden Bände ${ }^{2}$ geben das

1 So Ernst von Hippel, Archiv für Rechts- und Wirtschaftsphilosophie, Bd. XXIV (1930/31), H. 4, S. 271.

2 Zusammen mit dem ersten Band: Politik und Gesellschaft im Kaiserreich, herausgegeben und eingeleitet von Lothar Albertin, Tübingen 2007. 
politische und juristische Euvre des Autors bis 1918 mit jeweils circa 50 Stücken wieder, Band III steht noch aus, und der 2008 erschienene Band IV ist der Weimarer Republik gewidmet $^{3}$, während der letzte Band für die beiden vorgenannten Zeitphasen kommunalwissenschaftliche und -politische Beiträge zusammenfasst. ${ }^{4}$

Die vorliegende Sammlung lässt sich zunächst unter dem Aspekt der Greifbarkeit als eine Art Wiedergutmachung begrüßen, denn sie bündelt etliche Aufsätze, Stellungnahmen, Besprechungen usw., die bislang unbeschadet einer ersten Teilsammlung von $1926^{5}$ nur sehr verstreut und weiter durch die Bücherverbrennungen von 1933 und daran anschließende NS- wie kriegsbedingte Bibliotheksbereinigungen beziehungsweise -verluste nur noch dezimiert vorhanden sind.

Auf einer zweiten Ebene besteht das spezielle Verdienst von Band II darin, dass er gegenüber der letztlich konservativen Dominanz bis 1918 die damit konkurrierende, realgeschichtlich leider weniger wirkmächtige Gegenströmung eines facettenreichen Liberalismus in herausragender Weise ins Gedächtnis ruft, und zwar hinsichtlich einschlägiger politischer wie staatsrechtlicher Vorstellungen. Band wie Bände zu diesem bedeutenden und übrigens glänzend formulierenden deutschen Whig sind damit würdige Nachbarn der bereits länger vorhandenen entsprechenden Editionen zu großen Liberalen wie dem stärker visionären Friedrich Naumann und dem erratischen Max Weber. ${ }^{6}$ Gegenüber der im Kaiserreich von Paul Laband angeführten herrschenden Meinung zum Staatsrecht wird mit Preußeine namhafte Stimme aus dem Feld damaliger Mindermeinungen eindrucksvoll präsentiert. Dabei geht es einmal um Positionen auf Grundlage der von Georg Beseler begründeten und von seinem Schüler Otto Gierke ausgebauten Genossenschaftslehre. Diese eröffnete gegenüber den Realitäten des deutschen monarchischen Konstitutionalismus eine moderate Modernisierung, indem sie zwischen den Polen der auf konservativer Seite bevorzugten romantischorganologischen Staatsauffassung einerseits und einer aufklärerisch-liberalen assoziativen Staatsgestaltbarkeit andererseits einen Monarchie wahrenden Kompromiss bot. ${ }^{7}$ Sie zeigte damit einen Umbau vom monarchischen Anstalts- oder Obrigkeitsstaat hin zu einer stärker körperschaftlichen beziehungsweise volksstaatlichen Richtung auf. Insofern dokumentiert die vorliegende Sammlung verfassungsrechtliche Positionen, die alternativgeschichtlich bei nicht nur kurzzeitiger Kaiserschaft von Friedrich III, etwa bei einer seinerzeit aus konserva-

3 Zu Weimar vgl. Band III: Verfassungsentwürfe, Verfassungskommentare (in Vorbereitung); Band IV: Politik und Verfassung in der Weimarer Republik, herausgegeben und eingeleitet von Detlef Lehnert, Tübingen 2008.

4 Vgl. Band V: Kommunalwissenschaft und Kommunalpolitik, herausgegeben und eingeleitet von Christoph Müller, Tübingen 2012 (in Vorbereitung).

5 Vgl. Hugo Preuß, Staat, Recht und Freiheit. Aus 40 Jahren deutscher Politik und Geschichte, mit einem Geleitwort von Theodor Heuss, Tübingen 1926 (ND Hildesheim 1964 und 2010), wobei die darin gebrachten Beiträge sämtlich in der hiesigen Edition wieder erscheinen.

6 Vgl. Friedrich Naumann, Politische Schriften, in: ders., Werke, Bd. II bis V, herausgegeben von Theodor Schieder, Köln / Opladen 1964 (Neuausgabe in Vorbereitung); Max Weber, Gesamtausgabe, insbesondere Bd. I /3, 4, 15 - 17 und Bd. II / 5 - 9, Tübingen 1984-2008. Zu beiden Autoren bestand übrigens ausweislich der jeweiligen Inbezugnahmen kein besonderes Näheverhältnis.

7 Dazu näher Jörg-Detlef Kühne, Die Bedeutung der Genossenschaftslehre für die moderne Verfassung, in: ZParl, 15. Jg. (1984), H. 4, S. 552 - 570 sowie speziell zum Einpassungsproblem unter der Weimarer Verfassung Kathrin Groh, Demokratische Staatsrechtslehrer in der Weimarer Republik, Tübingen 2010, S. 19 ff. und passim. 
tiver Sicht ${ }^{8}$ befürchteten liberalen Reichsregierung Eduard Lasker / Albert Hänel, chancenreicher gewesen wären. Die vorliegenden Schriften machen jedenfalls deutlich, wie intensiv der Autor verfassungspolitisch und -rechtlich auf seine Rolle als maßgeblicher Verfassungsschöpfer für und in Weimar 1919 vorbereitet war.

Gleichzeitig ist zu sagen, dass Preuß entgegen jüngst geäußerter Auffassung ${ }^{9}$ weder politisch noch juristisch ein Grenzgänger oder Außenseiter gewesen ist. Vielmehr stand er weitestgehend innerhalb des breiten Bandes von Vorstellungen seinerzeitiger liberaler Juristen. Abgesehen von persönlichen Anwürfen antisemitischer Provenienz, ist für ihn wissenschaftlich im Wesentlichen nur eine sachliche Sonderposition auszumachen, nämlich die von ihm partizipatorisch weiterentwickelte Genossenschaftslehre, in der er an die Stelle klassischer die Rechts-Souveränität treten lässt, ohne indessen nennenswerte Gefolgschaft zu finden. Doch braucht diese staatstheoretische Konzeption unter dem thematischen Vorzeichen der ZParl nicht vertieft zu werden. Hier ist vielmehr von Interesse, dass Preuß schon vor 1919 politisch-praktisch agierte, und zwar als liberaler Berliner Stadtverordneter seit 1895 sowie als unbesoldetes Magistratsmitglied ab 1910. Wenngleich seine zusätzlichen Kandidaturen in den Reichstagswahlen von 1907 und 1912 erfolglos blieben ${ }^{10}$, erscheint mithin das Verdikt späterer DDP-Parteifreunde wie seines Weimarer Mitarbeiters Willibalt Apelt sowie von Erich Koch-Weser überzogen, er sei Theoretiker und kein praktischer Staatsmann gewesen. ${ }^{11}$ Wie politisch Preußdachte und sich äußerte, zeigen neben den in Band I der Edition abgedruckten Beiträgen auch die vorliegenden juristischen Stücke, deren Themenauswahl sein sicheres Gespür für die offenen Wunden im kaiserzeitlichen Staatsrecht verrät. Unmissverständlich sei dazu indessen sogleich ergänzt, dass seine rechtlichen Einzelausführungen durchaus theoriebewusst „völlig von allen politischen Gründen ab(zu)sehen“" (S. 366) bemüht sind. Dies ist allerdings nur Auftakt für den näher zu behandelnden Gewinn, den die verschiedenen Schriften bieten. So sei etwa methodologisch angeführt, dass Preuß bei seinen juristischen Deduktionen bereits früh und dezidiert das ansonsten erst deutlich später beachtete Interpretationsprinzip der „Einheit der Verfassung“ postuliert (S. 389); und weiter, dass er bei aller Neigung, entgegen Laband auch umfassend historische Interpretationsaspekte einzubeziehen, für entsprechende Feststellungen im Gesetzgebungsbereich eine treffliche, nach wie vor gültige Beschreibung der Schwierigkeiten gibt, aus der parlamentarischen Debatte klare Auskünfte zu gewinnen (S. 367).

Doch begibt man sich damit in rechtstheoretische wie -historische Bereiche, zu denen generell auf die intensive Einleitung von Dian Schefold (insbesondere S. 35 ff.) verwiesen sei. Vor weiterem Eingehen darauf ist festzuhalten, dass die Preußschen Texte $(\mathrm{Nr} .1$ - 49) in

8 So Heinrich von Treitschke, Brief an seinen Verleger vom 2. Februar 1882, in: ders., Briefe, herausgegeben von Max Cornicelius, Bd. III, Leipzig 1920, S. 536 f.

9 Vgl. Kathrin Groh, a.a.O. (Fn. 7), S. 19 ff.

10 Näher Carl-Wilhelm Reibel, Handbuch der Reichstagswahlen 1890-1918, Düsseldorf 2007, Nr. 81, Nr. 369.

11 So Willibalt Apelt, Jurist im Wandel der Staatsformen, Tübingen 1965, S. 84; ähnlich Erich KochWeser, Tagebucheintrag vom 13. Juni 1919, in s. Nl. (Bundesarchiv N 1012, Nr. 13): „Preuß hat die Erfahrung eines Politikers nicht gehabt.“ Anderer Auffassung demgegenüber Conrad Haußmann als Vorsitzender des Verfassungsausschusses in der Deutschen Nationalversammlung 1919, in: Verhandlungen des Deutschen Reichstags, Bd. 328, S. 2081 (69. Sitzung vom 29. Juli 1919); Richard Thoma, Besprechung des oben genannten Werks (Fn. 5), in: Schmollers Jahrbuch, 52. Jg. (1928), S. 943, S. 945, der dort die staatsmännische Qualität von Preuß hervorhebt. 
vier Abteilungen gruppiert sind, und zwar unter den hier verkürzt wiedergegebenen Überschriften: (1.) Theorie, (2.) völker-, staats- und verwaltungsgeschichtliche Einzelfragen, (3.) Verwaltungsreform und (4.) Rezensionen. Damit ergibt sich nur ein erster materieller, allerdings hinsichtlich der letztgenannten Abteilung nicht vollkonsistenter Gliederungszugriff. Die tiefdringende Einleitung von Schefold hat sich deshalb von diesem Viererschema wie in den vorausgehenden Bänden des Gesamtwerks richtigerweise gelöst und widmet sich den Schriften dieses Bandes unter circa 30 Stichpunkten.

Es liegt auf der Hand, dass es an dieser Stelle primär darum geht, sich auf solche Schriften von Preuß zu konzentrieren, die das damalige Regierungssystem samt Stellung und Wirkungsmöglichkeiten des Parlaments behandeln. Dazu lässt sich auf den Spuren der Einleitung und sie teilweise ergänzend Folgendes hervorheben: Zum einen ist es die liberale politische Sozialisation von Preuß, die ihm als politischem Professor spezifisches juristisches Profil gibt und der Annahme seiner Grenzgänger- oder Außenseiterrolle wiederholt widerstreitet, denn er zeigt sich deutlich den Vorstellungen und Anliegen der Früh- und Altliberalen wie Freiherr vom Stein, Georg Beseler und Otto (von) Gierke sowie den gouvernementalen Nationalliberalen Johannes Caspar Bluntschli und Rudolf(von) Gneist wie auch den eher partizipatorischen Fortschritts- oder Linksliberalen wie Albert Hänel und Franz von Holtzendorff verpflichtet. Vor diesem Hintergrund ist nicht nur sein Einsatz für eine stärker partizipatorische Entfaltung der Genossenschaftslehre zu sehen, sondern auch seine Befassung mit Parlamentsrechten, und zwar speziell bei Militärhaushalt (Nr. 17) und Ministerverantwortlichkeit (Nr. 20). Dem entspricht weiter seine, entgegen der damaligen stärker monarchisch geprägten herrschenden Meinung, selbstverständliche Behandlung des Reichstags als einem ebenfalls obersten Verfassungsorgan (Nr. 26) sowie sein klares rechtsstaatliches Defizitbewusstsein hinsichtlich fehlender Verfassungsgerichtsbarkeit (Nr. 27). Auch ehrt es Preußals betont rechtsstaatlich gesonnenen Liberalen, dass er das preußische Dreiklassenwahlrecht, trotz dessen politischer Begünstigung liberaler Kräfte, als ungleich beanstandet (S. 561 f.) und ebenso das Reichstagswahlgesetz kritisiert (S. 558). Letzteres wurde bekanntermaßen trotz zunehmender Verstädterung und damit einhergehender Gleichheitsverzerrung der Wahlkreisgrößen zu Lasten der Linken vom Gros des Reichstags samt seinen liberalen Fraktionen bis 1918 unverändert belassen. Hinzu kommt die Behandlung des bis heute in seiner Wirksamkeit unaufgearbeiteten staatlichen Bestätigungsrechts hinsichtlich der Wahlbesetzung kommunaler Spitzenämter (Nr. 25), was erneut den wachen Blick des Autors für offene Wunden des damaligen Konstitutionalismus belegt.

Dass Preuß weiterhin in seiner rechtlichen Argumentation stets lege artis arbeitet und dabei keine Extrem- oder Außenseiterpositionen bezieht, dafür seien beispielhaft seine Deduktionen zum Budgetrecht des Reichstags in Militärfragen angeführt. Er schließt im Ergebnis nämlich keineswegs zur Dauerforderung der politischen Altliberalen auf, wie für den Zivil- auch für den Militärhaushalt den Annuitätsgrundsatz durchzusetzen (S. 366). Und ebenso bleibt seine Besprechung der bekannteren Kommentierung der preußischen Grundrechte durch Anschütz (Nr. 28) bei aller Kritik (S. 574 ff.) hinter der rechtlich grundsätzlicheren, auf märzliberale Ursprünge zurückgehenden Auseinandersetzung mit demselben Werk durch Richard Thoma ${ }^{12}$ zurück.

12 Richard Thoma, Der Vorbehalt des Gesetzes im preußischen Verfassungsrecht, in: Festgabe für Otto Mayer, Tübingen 1916 (ND Aalen 1964), S. 165, S. 214 f. 
Schließlich ragt Preuß dadurch sehr positiv hervor, dass ihm der eigentümliche Autismus, der in der damaligen deutschen Juristendiskussion internationale Rechtsvergleichung in erstaunlichem Ausmaß ausfallen lässt, nicht nachgesagt werden kann. Vielmehr gehört er wie sein Lehrer Rudolf(von) Gneist, wie Georg Jellinek und dessen Schüler Julius Hatschek zur verhältnismäßig kleinen Gruppe deutscher Rechtswissenschaftler, die bereits damals Rechtserkenntnis über die Staatsgrenzen hinaus sucht. Bezeichnend ist dafür seine mehrfache Befassung mit Franz (Francis) Lieber (Nr. 5 f., Nr. 21), der als Teilnehmer der Befreiungskriege später in die Vereinigten Staaten emigrierte, dort bedeutender Staatswissenschaftler wurde und wie bereits 1848/49 in engem wissenschaftlichen Austausch mit deutschen Liberalen blieb. Man beachte weiter die rechtsvergleichenden Arbeiten von Preuß zur Verwaltungsund Staatsreform in Österreich und Preußen (Nr. 32) und zum englischen Selfgovernment (S. 241 ff.). Die Spannweite seines vergleichenden Ausgriffs geht von der amerikanischen Implied-powers-Doktrin (S. 773) bis hin zum Schweizer Milizsystem (S. 399) und erfasst unter anderem Locke, Montesquieu wie Rousseau.

Zusammenfassend vermittelt gerade die kompakte Greifbarkeit der vorliegenden Stücke einen vorzüglichen Eindruck darüber, welche juristischen Potenziale im Bereich des seinerzeitigen Liberalismus vorhanden waren. Wenngleich sie in der Kaiserzeit nicht zu vollem Durchbruch gelangten, wirkten sie ab 1918. Insofern unterstreicht das Vorwort der Herausgeber (S. VII) zu Recht, wie intensiv Preuß hinsichtlich der großen, ihm 1919 angetragenen Arbeit für die Weimarer Reichsverfassung vorbereitet war. Im Geleitwort des Bundestagspräsidenten (S. V) wird darüber hinaus zutreffend der Leistung Anerkennung gezollt, dass der vorliegende Band samt der Gesamtedition weiterem Erinnerungsverlust vorbeugt. Es ist insbesondere die Ideengeschichte, die damit hervortritt und als ein Kernbestandteil der Verfassungshistorie diese authentisch zurechtzurücken geeignet ist: insgesamt ein grandioser Grundlagenimpuls in Richtung demokratischer Verfassungsgeschichte im deutschen Kulturraum. Die hier versammelten Schriften bieten, neben den bereits erwähnten Sammlungen von Naumann und Weber ${ }^{13}$, ein weiteres massives Beispiel für das Ringen um Demokratisierung und Parlamentarisierung im damals breiten Strom des deutschen Liberalismus. Innerhalb dieser Trias zeigt sich Preuß gegenüber dem staatspolitischen Euvre von Naumann deutlich juristischer. Und gegenüber dem von Weber und dessen Anflügen eines bürgerlichen Imperialismus ${ }^{14}$ besticht er durch eine für seine Zeit bemerkenswerte, völlig unmartialische, auch während des Ersten Weltkriegs aufrecht erhaltene Zivilität. ${ }^{15}$ In einer Gegenwart ubiquitär anwachsender militärischer Einsatzbereitschaft, bei der bedauerlicherweise wieder über Präventivschläge und Euphemismen wie „Elitesoldaten“ schwadroniert wird, kann diese - in der Blütezeit des Imperialismus - vorbildliche Nüchternheit nicht zuletzt Ansporn wie Ermutigung sein.

Jörg-Detlef Kühne

13 Dazu siehe Fußnote 6.

14 So vor allem in seiner Freiburger Antrittsvorlesung von 1895 „Der Nationalstaat und die Volkswirtschaftspolitik“, in: ders., Gesammelte politische Schriften, Tübingen 1988, S. 1 ff.

15 So enthielt sich Preuß der professoralen Kriegsaufrufe, zu den es insbesondere ab 1915 kam, vgl. Günther Gillessen, Hugo Preuß, Berlin 2000, S. 82, Fn. 1. 